\title{
Evaluación de la hidrólisis enzimática de wet white asistida con ultrasonido para obtener colágeno hidrolizado
}

\author{
Leidy Yaneth Almonacid-Jiménez ${ }^{1}$ \\ Juan Sebastián Vallejo-Rodríguez ${ }^{2}$ \\ Rafael Nikolay Agudelo-Valencia ${ }^{3}$ \\ Javier Adolfo Hernández-Fernández ${ }^{4}$ \\ Óscar Leonardo Ortiz-Medina ${ }^{5}$ \\ Diana Paola Ovalle-González ${ }^{6}$
}

Fecha de recepción: 12 de febrero de 2019

Fecha de aceptación: 21 de junio de 2019

\section{Resumen}

La presente investigación se centra en la evaluación del método de hidrólisis enzimática asistida por ultrasonido en la obtención de colágeno hidrolizado a partir del aprovechamiento del residuo (wet white) proveniente del cuero curtido con glutaraldehído, con la finalidad de minimizar la generación de impactos ambientales por dicho material. Se evaluó el efecto del pH de la solución, dosis de enzima, intensidad de ultrasonido aplicado y temperatura de la mezcla, manteniendo constantes la masa inicial de wet white, el volumen de agua y el tiempo de reacción. Los resultados experimentales señalan que para pH 9 dosis de enzima Tan G Plus $0.00012 \mathrm{~kg}$, temperatura de $313.15 \mathrm{~K}$ e intensidad de ultrasonido del $40 \%$ se logra degradar el material y desnaturalizar la proteína, obteniendo una concentración en

\footnotetext{
1 Universidad Libre (Bogotá-Distrito Capital, Colombia). leidyy-almonacidj@unilibre.edu.co. ORCID: 0000-00033167-3737.

$\frac{31}{2}$ Universidad Libre (Bogotá-Distrito Capital, Colombia). juans-vallejor@unilibre.edu.co. ORCID: 0000-00022290-2182.

${ }^{3}$ M. Sc. Universidad Libre (Bogotá-Distrito Capital, Colombia). rafaeln.agudelov@unilibre.edu.co. ORCID: 00000002-6646-7725

${ }^{4}$ Ph. D. (c) Universidad Jorge Tadeo Lozano (Bogotá-Distrito Capital, Colombia). ORCID: 0000-0001-84429266.

${ }^{5}$ Ph. D. Universidad Libre (Bogotá-Distrito Capital, Colombia). oscar.ortiz@unilibre.edu.co. ORCID: 0000-00015587-5654.

${ }^{6}$ Universidad Libre (Bogotá-Distrito Capital, Colombia). dianap-ovalleg@unilibre.edu.co. ORCID: 0000-00019832-1010.

Revista Facultad de Ingeniería (Rev. Fac. Ing.) Vol. 28 (52), pp. 59-75. Julio-Septiembre 2019. Tunja-Boyacá, Colombia. L-ISSN: 0121-1129, e-ISSN: 2357-5328, DOI:

https://doi.org/10.19053/01211129.v28.n52.2019.9654
} 
peso de colágeno hidrolizado de $3.013 \mathrm{~kg} / \mathrm{m}^{3}$, por cada kilogramo de material empleado, el cual representa una recuperación del $3.0 \%$ en masa de wet white. Se observa que las variables más representativas para el proceso analizado son el pH y la temperatura, que dan un mayor rendimiento al proceso y permiten obtener mayor cantidad de proteína hidrolizada.

Palabras clave: colágeno; hidrólisis enzimática; impactos ambientales; ultrasonido; wet white.

\section{Evaluation of the Enzymatic Hydrolysis of Wet White Assisted with Ultrasound to Obtain Hydrolyzed Collagen}

\section{Abstract}

The present investigation focuses on the evaluation of the enzymatic hydrolysis method assisted by ultrasound in obtaining hydrolyzed collagen from the use of the residue (wet white) from leather tanned with glutaraldehyde, in order to minimize the generation of environmental impacts that have occurred for such material. The effect of $\mathrm{pH}$ of the solution, enzyme dose, intensity of applied ultrasound, and temperature of the mixture were evaluated, keeping constant the initial mass of wet white, volume of water and reaction time. The experimental results indicate that for $\mathrm{pH} 9$ doses of enzyme Tan G Plus $0.00012 \mathrm{~kg}$, temperature of $313.15 \mathrm{~K}$ and intensity of ultrasound of $40 \%$ it is possible to degrade the material and denature the protein, obtaining a hydrolyzed collagen weight concentration of $3.013 \mathrm{~kg} / \mathrm{m}^{3}$, for each kilogram of material used, which represents a $3.0 \%$ mass recovery of wet white. It is observed that the most representative variables for the analyzed process are the $\mathrm{pH}$ and the temperature, giving a higher performance to the process allowing get a higher amount of hydrolyzed protein.

Keywords: collagen; environmental impacts; enzymatic hydrolysis; ultrasound; wet white. 


\section{Avaliação da hidrólise enzimática de wet white assistida com ultrassom para obter colágeno hidrolisado}

\section{Resumo}

A presente pesquisa centra-se na avaliação do método de hidrólise enzimática assistida por ultrassom na obtenção de colágeno hidrolisado a partir do aproveitamento do resíduo (wet white) proveniente do couro curtido com glutaraldeído, com a finalidade de minimizar a geração de impactos ambientais por tal material. Avaliou-se o efeito do $\mathrm{pH}$ da solução, dose de enzima, intensidade de ultrassom aplicado e temperatura da mistura, mantendo constantes a massa inicial de wet white, o volume de água e o tempo de reação. Os resultados experimentais apontam que para pH 9 dose de enzima Tan G Plus $0.00012 \mathrm{~kg}$, temperatura de $313.15 \mathrm{~K}$ e intensidade de ultrassom de $40 \%$, logra-se degradar o material e desnaturalizar a proteína, obtendo uma concentração em peso de colágeno hidrolisado de $3.013 \mathrm{~kg} / \mathrm{m}^{3}$, por cada quilograma de material empregado, o qual representa uma recuperação de $3.0 \%$ em massa de wet white. Observa-se que as variáveis mais representativas para o processo analisado são o pH e a temperatura, que dão um maior rendimento ao processo e permitem obter maior quantidade de proteína hidrolisada.

Palavras chave: colágeno; hidrólise enzimática; impactos ambientais; ultrassom; wet white.

\footnotetext{
Para citar este artículo:

L. Y. Almonacid-Jiménez, J. S. Vallejo-Rodríguez, R. N. Agudelo-Valencia, J. A. HernándezFernández, Ó. L. Ortiz-Medina, and D. P. Ovalle-González, "Evaluación de la hidrólisis enzimática de wet white asistida con ultrasonido para obtener colágeno hidrolizado," Revista Facultad de Ingeniería, vol. 28 (52), pp. 59-75, Jul. 2019 https://doi.org/10.19053/01211129.v28.n52.2019.9654.
}

Esta obra está bajo licencia internacional Creative Commons Reconocimiento 4.0

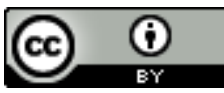




\section{INTRODUCCIÓN}

El sector industrial curtidor es considerado como uno de los que más ocasionan contaminación ambiental [1], debido a que los procesos de transformación del cuero para la elaboración de marroquinería generan altas cargas de residuos sólidos, líquidos y gaseosos [2]. Esto trae consigo la necesidad de desarrollar y emplear tecnologías limpias y sostenibles que reduzcan la contaminación causada por la actividad industrial [3] y así disminuir los impactos económicos, sociales y ambientales, debido a que los residuos sólidos y líquidos afectan considerablemente la calidad ambiental del ecosistema [2]. Por tal motivo se busca involucrar alternativas para la minimización o prevención de la contaminación por medio de proyectos de producción más limpia para dicha industria [4]. Los municipios de Villapinzón y Chocontá, del departamento de Cundinamarca, Colombia, poseen bodegas rudimentarias, curtiembres 0 tenerías en los alrededores del río Bogotá, con el fin de disponer del agua para el proceso o como medio de evacuación de los vertimientos generados dentro del proceso.

Durante el proceso de curtido se producen residuos, como viruta de wet white, residuos de viruta de cuero curtido (wet blue) y subproductos como recortes de piel, carnaza, unche, entre otros [5]. El curtido wet white (WW) es elaborado en la etapa de rebajado de pieles, con glutaraldehído principalmente, agentes aldehídicos, taninos vegetales y sintéticos o con minerales como sales de circonio y aluminio [6], donde, de acuerdo con algunos estudios, el wet white con glutaraldehído resulta ser más efectivo, pues da lugar a la obtención de residuos con mayor facilidad de disposición final y tratamiento que el realizado de forma convencional, lo cual representa menor riesgo para el ambiente, dado que el proceso no tiene metales no esenciales [7, 8]. El proceso de curtido libre de cromo, wet white, se ha llevado a cabo en la industria curtidora con el propósito de emplear nuevas alternativas innovadoras en la fabricación del cuero ecológico libre de cromo, biodegradable y con las mismas características del realizado habitualmente [9], a fin de reducir los niveles de contaminación, cumplir con los requerimientos ambientales y contribuir al mejoramiento continuo del ambiente [10]. Sin embargo, al no desechar o dar el 
tratamiento pertinente a dichos residuos se puede presentar una problemática ambiental, por lo cual se estudia el tratamiento de las virutas de wet white.

La composición de colágeno representa el $29 \%$ del $33 \%$ de las proteínas estructurales totales presentes en la piel de origen bovino, de las cuales el $2 \%$ es keratina y el $0.3 \%$, elastina [11]. Por tal razón, es adecuado investigar sobre la extracción de colágeno hidrolizado, el cual es una proteína que brinda estructura, elasticidad, fuerza y forma a los tejidos extracelulares conectores [15], se presenta en los residuos y/o subproductos generados dentro del proceso de curtido y es aplicado en diferentes campos como en medicina, nutrición, cosméticos y fármacos [16], como la elaboración de productos de belleza y cuidado de la piel [12], es decir, champú, acondicionadores, lociones, cremas [13], jabones, cepillos, pinceles, fabricación de alimentos para animales [14], pastas, entre otros productos.

En la actualidad existen alternativas de aprovechamiento y valorización del material por tratar, como la obtención de hidrolizados de colágeno; alternativas que se han enfocado en el tratamiento de las virutas de cuero curtido wet blue a través del proceso de hidrólisis alcalina-enzimática [17, 18]. De igual forma, de los residuos de curtido libre de cromo se extraen virutas (wet white) que pueden ser aprovechadas para la producción de proteínas hidrolizadas, lo cual valoriza el residuo y mitiga el impacto generado, además de favorecer la sostenibilidad económica de la industria curtidora, debido a que puede ser una fuente adicional de recursos del sector. La hidrólisis enzimática asistida con ultrasonido representa una alternativa para la intensificación del proceso de fabricación de proteínas hidrolizadas a partir de residuos generados por el sector curtidor, el cual procura reducir el uso de agentes químicos por efecto de la mejoría en la agitación [19], acelerar el proceso y obtener un mayor rendimiento en la extracción [20, 21]. El proceso se orientó a evaluar una opción para mejorar la productividad de la industria curtidora a partir de la obtención de colágeno hidrolizado como subproducto del proceso. 


\section{Metodología}

\section{A. Materiales}

Para la ejecución de los ensayos experimentales en la obtención de colágeno hidrolizado la materia prima fueron virutas de wet white suministrada por una empresa curtidora ubicada en el municipio de Villapinzón. La enzima proteolítica utilizada tiene el nombre comercial Tan G Plus, provista por la empresa MerQuiand, distribuidora de enzimas de la marca Dupont, Gerencor International, empleadas comúnmente en la industria curtidora [20] y en la hidrólisis de proteínas. Puntualmente es seleccionada en la presente investigación debido a que permite el desprendimiento de las fibras de colágeno [21]. Por otro lado, los ensayos de hidrólisis fueron realizados en el baño ultrasónico Wise Clean modelo WUC-D10H de la marca Wisd Laboratory con capacidad de $0.01 \mathrm{~m}^{3}$, temporizador hasta 216.000 segundos, alta frecuencia de $40 \mathrm{kHz}$, control y rangos de temperatura hasta 353.15 K [22]; dentro del baño fueron dispuestos beakers de vidrio con capacidad de $0.00025 \mathrm{~m}^{3}$, cada uno contenía $0.01 \mathrm{~kg}$ de material medido con la balanza analítica, $0.0001 \mathrm{~m}^{3}$ de agua destilada a pH de operación y la cantidad de enzima determinada para la ejecución de los ensayos, equipos dispuestos por los laboratorios de la Universidad Libre sede Bosque Popular.

El diseño experimental implementado fue del tipo central compuesto [23], y consistió en la elaboración de 20 ensayos para los cuales las variables o factores de proceso fueron el pH de la mezcla reactiva, el cual varió entre 6 y 9, la dosis de la enzima TAN G PLUS entre $0.000075 \mathrm{~kg}$ y $0.00012 \mathrm{~kg}$ por cada $0.01 \mathrm{~kg}$ de virutas para hidrolizar, y la potencia del baño ultrasónico fue variada entre el 40 y el $60 \%$ de la máxima capacidad del equipo. Las variables fijas del proceso fueron la masa de material $0.01 \mathrm{~kg}$, el volumen de agua para la reacción $0.0001 \mathrm{~m}^{3}$ y el tiempo de hidrólisis 14.400 segundos. Por otro lado, la variable de respuesta fue la masa de colágeno hidrolizado obtenido. Una vez determinadas las condiciones óptimas de funcionamiento, se realizaron ensayos a diferentes temperaturas y por triplicado, variadas entre $298.15 \mathrm{~K}, 303.15 \mathrm{~K}, 308.15 \mathrm{~K}$ y $313.15 \mathrm{~K}$, con el propósito de 
encontrar el efecto de la temperatura sobre la eficiencia en la obtención de colágeno a partir de virutas de wet white.

\section{B. Métodos}

El colágeno hidrolizado se obtuvo por hidrólisis enzimática asistida por ultrasonido [19], a partir de virutas de cuero curtido con glutaraldehído (wet white). La cuantificación de colágeno hidrolizado fue realizada por el método de Bradford [24] y electroforesis en gel de SDS-poliacrilamida [25], para verificar y reconocer la pureza de los sustratos o soluciones de colágeno.

1) Caracterización de la proteína obtenida. La determinación de la concentración de proteína presente en las muestras a condiciones de $\mathrm{pH}$ y dosis de enzima experimentales en la obtención de colágeno hidrolizado se realizó mediante el método de Bradford [26]. Con la finalidad de verificar la extracción de colágeno a partir de virutas de wet white, se desarrolló la electroforesis en gel de poliacrilamida SDS-PAGE partiendo del método de Laemmli, el cual permite el reconocimiento de las proteínas en alta cantidad mediante la tinción [27]. Se implementó un gel de separación al $12 \%$ y concentrador al 4 \%; la preparación de las mezclas fue hecha a partir de Buffer Tris- $\mathrm{HCl} 1.5 \mathrm{M} \mathrm{pH} 8.8$, agua destilada, bis-acrilamida al $30 \%$, SDS al $30 \%$, persulfato de amonio (APS), TEMED, Buffer Tris- $\mathrm{HCl} 0.5 \mathrm{M} \mathrm{pH} 6.8$ respectivamente para cada uno de los geles. Para el corrido de las muestras, fueron sembradas en cada celda $20 \mu \mathrm{L}$ en el equipo Bio-Rad Mini-Protein tetra cell, se ajustó el voltaje a $125 \mathrm{~V}$. En cuanto a la determinación del tipo y pureza de la proteína hidrolizada (colágeno), se tomó como referencia el patrón Opti-Protein XL Marker con peso molecular de $245 \mathrm{kDa}$ [28].

2) Determinación del valor másico porcentual. La ecuación (1) permite ver el porcentaje de recuperación de proteína hidrolizada obtenida en los ensayos.

$$
\text { Valor Másico Porcentual }=\left(\frac{\text { Cantidad colágeno hidrolizado }\left(\frac{\mathrm{kg}}{\mathrm{m}^{3}}\right) * \text { Volumen de Agua }\left(\mathrm{m}^{3}\right)}{\text { Cantidad de Material Empleado }(\mathrm{kg})} * 100 \%\right)
$$


3) Análisis estadístico. El análisis estadístico de los resultados experimentales es desarrollado mediante la aplicación del método de análisis de varianza (ANOVA) [29] y el método de superficie de respuesta [23], que permiten la evaluación de las variables experimentales y las interacciones entre sí, para, de esa manera, determinar los factores que influyen directamente sobre la cantidad de colágeno hidrolizado obtenido y optimizar el proceso para alcanzar el mayor valor de la variable de interés o de respuesta.

\section{RESULTADOS}

Con el propósito de evaluar dicho proceso, en la Tabla 1 se encuentra el Anova para los experimentos realizados.

Tabla 1. Análisis de varianza de los experimentos

\begin{tabular}{|c|c|c|c|c|c|}
\hline Fuente de variación & $\begin{array}{c}\text { Suma de } \\
\text { cuadrados }\end{array}$ & $\begin{array}{c}\text { Grados de } \\
\text { libertad }\end{array}$ & $\begin{array}{c}\text { Cuadrados } \\
\text { medios }\end{array}$ & Valor F & Prob > F \\
\hline Modelo & 3.396 & 8 & 0.4245 & 26.808 & $<0.0001$ \\
\hline $\mathrm{A}: \mathrm{pH}$ & 1.845 & 1 & 1.8447 & 116.488 & $<0.0001$ \\
\hline $\mathrm{B}:$ Dosis de enzima & 0.220 & 1 & 0.2204 & 13.918 & 0.0033 \\
\hline C: intensidad de ultrasonido & 0.012 & 1 & 0.0123 & 0.776 & 0.3972 \\
\hline $\mathrm{A}^{2}$ & 0.094 & 1 & 0.0940 & 5.935 & 0.0331 \\
\hline $\mathrm{AB}$ & 0.161 & 1 & 0.1609 & 10.162 & 0.0086 \\
\hline $\mathrm{AC}$ & 0.234 & 1 & 0.2341 & 14.784 & 0.0027 \\
\hline $\mathrm{BC}$ & 0.136 & 1 & 0.1356 & 8.560 & 0.0138 \\
\hline $\mathrm{ABC}$ & 0.067 & 1 & 0.0669 & 4.227 & 0.0643 \\
\hline Residual & 0.174 & 11 & 0.0158 & & \\
\hline Falta de ajuste & 0.174 & 4 & 0.0435 & & \\
\hline Error puro & 0.000 & 7 & 0.0000 & & \\
\hline Total corregido & 3.570 & 19 & & & \\
\hline
\end{tabular}

Los valores de probabilidad $<0.0001$ señalan que las variables $\mathrm{pH}$ y dosis de enzima son las más significativas al extraer colágeno hidrolizado. Hecho que permite afirmar que los resultados de los ensayos efectivamente varían dependiendo de los valores de las variables o factores analizados, pero, por otra parte, el efecto de la intensidad del ultrasonido no representa un rol significativo que altere el rendimiento del proceso. 


\section{A. Influencia de las variables de reacción}

De los resultados experimentales se determinó que el pH y la dosis de enzima Tan G Plus, son las variables con mayor influencia sobre la cantidad de colágeno hidrolizado. La Figura 1 permite observar la interacción pH y dosis de enzima, de modo que para la dosis más alta se logra el mayor rendimiento a pH alcalino [30]. Se aprecia que las variables o factores estudiados interactúan levemente a partir de $\mathrm{pH}$ cercano a 8, punto en el cual al aumentar la dosis de enzima aumenta la concentración de proteína en la mezcla reactiva.

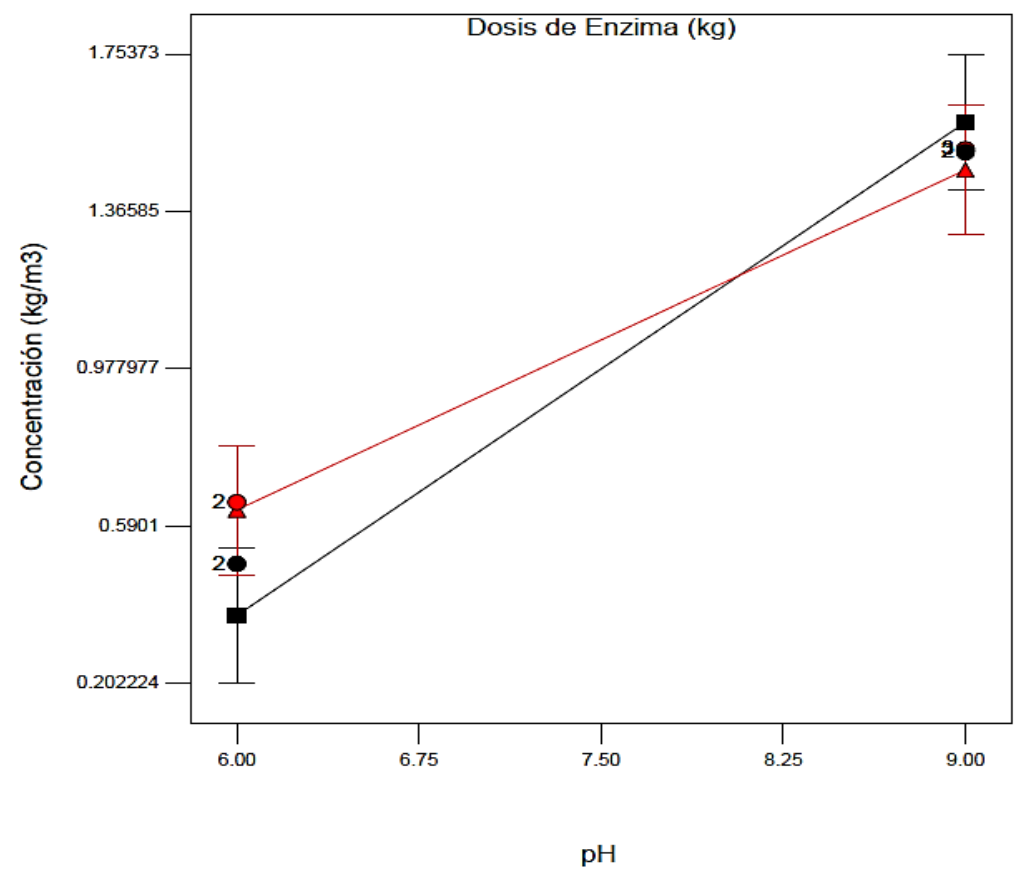

Fig. 1. Influencia de las variables de reacción en la obtención de colágeno hidrolizado.

La Figura 2 corresponde a la superficie de respuesta para los experimentos realizados en un tiempo de reacción de 14.400 segundos. 


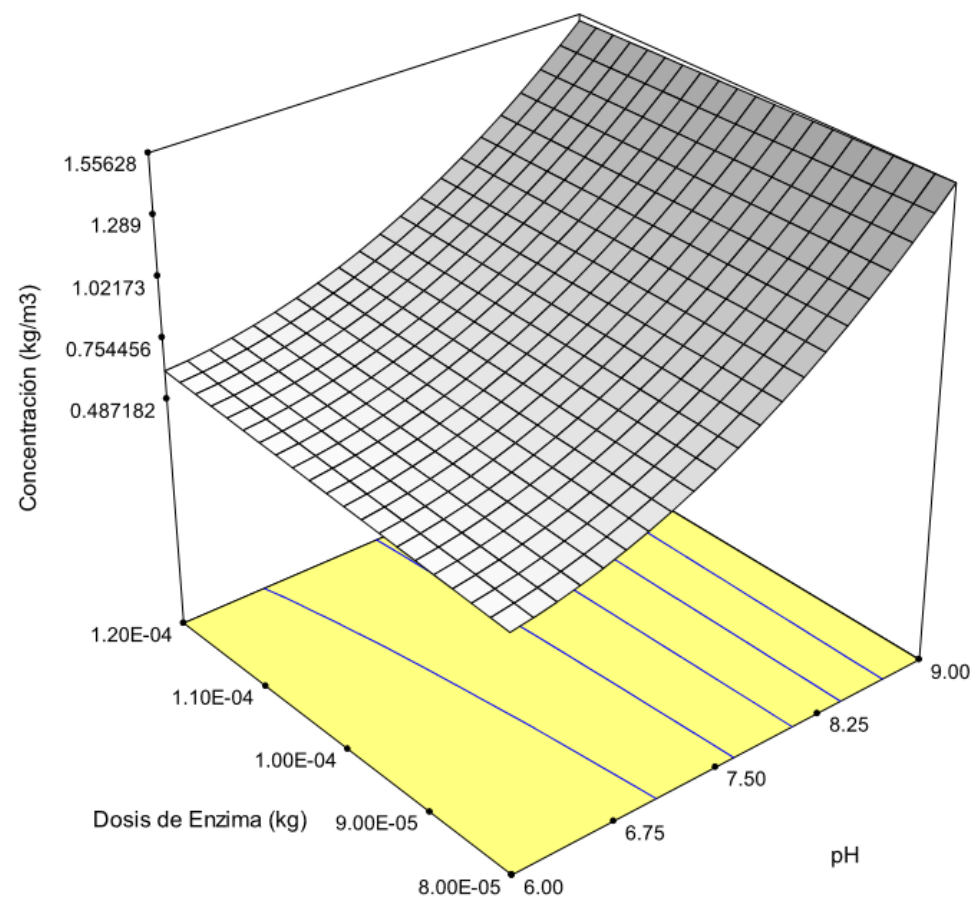

Fig. 2. Condiciones óptimas de reacción.

Se advierte en la figura que, a una misma dosis de enzima, el incremento de $\mathrm{pH}$ mejora la efectividad de la hidrólisis; por otra parte, el aumento de la dosis de enzima para $\mathrm{pH}$ constante no representa una mejoría significativa en el proceso hidrolítico, en términos de concentración de proteína hidrolizada extraída. Además, la intensidad de ultrasonido en la obtención de la proteína hidrolizada no representó una variación significativa en el proceso analizado; sin embargo, para el valor más bajo de la potencia (40\%) resultó ser la variable óptima, en conjunto con las variables $\mathrm{pH}$ 9, dosis de enzima $0.00012 \mathrm{~kg}$ y tiempo de reacción de 14.400 segundos, que permiten obtener $1.519 \mathrm{~kg}$ de proteína $/ \mathrm{m}^{3}$ de mezcla a temperatura ambiente. La ecuación (2) corresponde a la superficie de respuesta para la combinación de variables analizada:

Concentración $=8.32789-1.42886 * p H-80279.49971 *$ Dosis de enzima $0.15228 *$ Frecuencia de ultrasonido $+0.088163 * p H 2+8179.93469 * p H *$ Dosis de enzima $+0.014155 * p H *$ Frecuencia de ultrasonido $+2279.82932 *$ 
Dosis de enzima $*$ Frecuencia de ultrasonido $-236.81279 * p H *$

Dosis de enzima * Frecuencia de ultrasonido.

El coeficiente de correlación $\left(\mathrm{R}^{2}\right)$ conseguido del modelo para la superficie de respuesta tiene un valor de 0.9512 , lo cual permite afirmar que el ajuste de los datos experimentales es aceptable. A partir de la ecuación anterior se determinó que para $\mathrm{pH} 9$, dosis $0.00012 \mathrm{~kg}$, potencia $40 \%$, se alcanza la máxima concentración de $1.519 \mathrm{~kg} / \mathrm{m}^{3}$ de la mezcla de valores de las variables o factores que permiten alcanzar la mayor cantidad de colágeno hidrolizado.

\section{B. Influencia de la temperatura (K)}

A fin de establecer el efecto de la temperatura sobre la obtención de proteína hidrolizada y agilizar el proceso de extracción [31], se llevó a cabo la hidrólisis a pH 9.0, dosis $0.00012 \mathrm{~kg}$ e intensidad de ultrasonido $40 \%$ para temperaturas de 298.15 $\mathrm{K}, 303.15 \mathrm{~K}, 308.15 \mathrm{~K}$ y $313.15 \mathrm{~K}$. Los resultados de concentración conseguida en estos ensayos se presentan en la Figura 3.

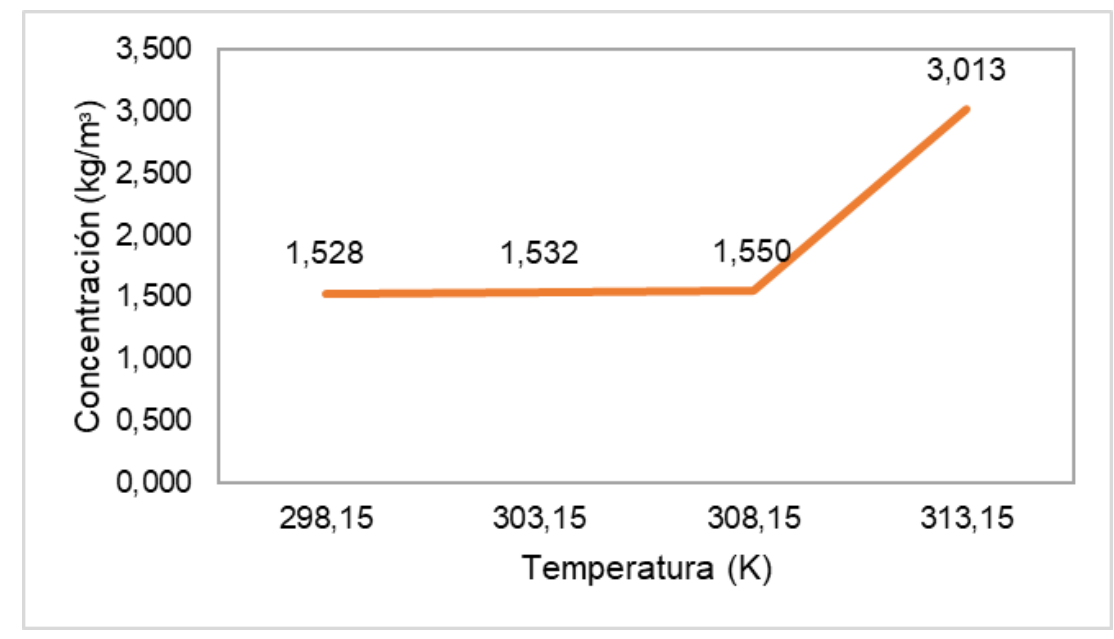

Fig. 3. Influencia de la temperatura en la obtención de colágeno hidrolizado.

En la Figura 3 se observa que al incrementar la temperatura, la cantidad de proteína hidrolizada aumenta a $313.15 \mathrm{~K}$ y se alcanza la mayor concentración en peso de 
colágeno hidrolizado, $3.013 \mathrm{~kg} / \mathrm{m}^{3}$, es decir, una recuperación del $3.0 \%$ de proteína hidrolizada por cada kilogramo de virutas de wet white empleado.

\section{Electroforesis en gel de poliacrilamida SDS-PAGE}

La Figura 4 corresponde al resultado de la electroforesis en gel SDS-PAGE, para la proteina hidrolizada extraída bajo las condicones óptimas determinadas a partir de la superficie de respuesta. El carril 1 de la figura corresponde al patrón empleado y el carril 2 representa la muestra de proteina hidrolizada. Se pueden señalar las bandas correspondientes a la proteína hidrolizada por encima del valor (245 kDa), lo cual confirma la presencia de colágeno hidrolizado, partiendo del hecho de que al colágeno hidrolizado corresponde una masa molecular de $300 \mathrm{kDa}$ [32], y con base en lo expuesto en otros estudios se puede afirmar que la proteína revelada en esta banda se debe a colágeno hidrolizado tipo I [25].

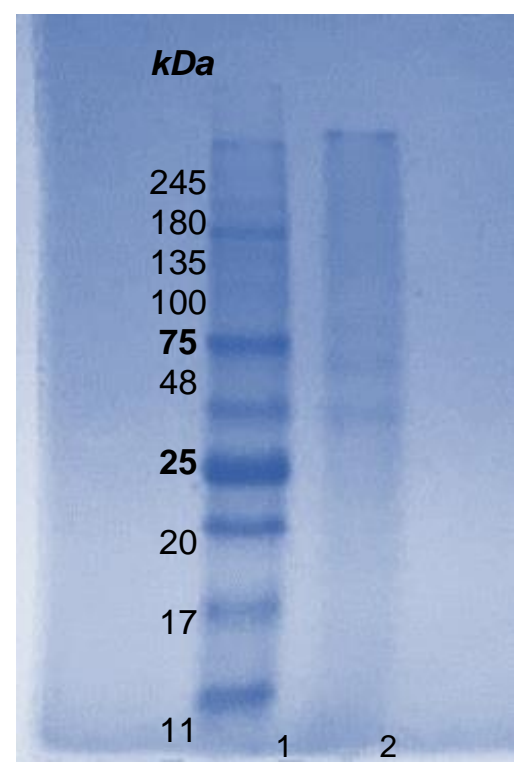

Fig. 4. Electroforesis en gel de poliacrilamida SDS-PAGE.

\section{DISCUSIÓN Y CONCLUSIONES}

En este estudio se demuestra que las variables de $\mathrm{pH}$ y dosis de enzima en su mayor valor permiten la obtención del colágeno en mayor proporción, donde el 
efecto de la intensidad de ultrasonido no es de gran importancia, debido a que supera los niveles de significancia presentes en el Anova. Por otro lado, la temperatura es una variable condicionante del proceso, ya que al aumentarse, se logra extraer mayor proteína. Sin embargo, debido a que las proteínas son susceptibles a altas temperaturas, estas pueden desnaturalizarse, razón por la cual no es recomendable aumentar más esta variable. De esta manera se logra evidenciar que las variables más significativas que permiten optimizar el proceso de hidrólisis enzimática para la fabricación de colágeno hidrolizado son el $\mathrm{pH}$, dosis de enzima y la temperatura. Además, se demuestra que el $\mathrm{pH}$ y la temperatura de operación se deben controlar y mantener dentro del mismo valor a lo largo del tiempo de operación.

Teniendo en cuenta que el proceso de curtido libre de cromo (wet white) es un procedimiento nuevo, no presenta estudios relacionados con la extracción de colágeno hidrolizado a partir de dicho material, por lo que se considera que el $3 \%$ del rendimiento es bueno y permite evidenciar que es posible su aprovechamiento. Dicho proceso se compara con estudios llevados a cabo en virutas de wet blue, ya que se obtienen de la misma etapa de rebajado, y se propone la fabricación del colágeno hidrolizado mediante la hidrólisis enzimática, donde se presenta un rendimiento del $4.5 \%$ con una mayor cantidad de material empleado. Por otra parte, en la electroforesis practicada se determinó la presencia de colágeno hidrolizado mediante la tinción de las bandas, donde los factores pueden afectar la coloración con mayor nitidez son el voltaje del equipo y el pH del medio.

\section{CONTRIBUCIÓN DE LOS AUTORES}

La contribución de los autores en la presente investigación se llevó a cabo con la ejecución de los ensayos experimentales y posterior análisis de resultados por Leidy Yaneth Almonacid Jiménez, Juan Sebastián Vallejo Rodríguez, Diana Paola Ovalle González y Rafael Nikolay Agudelo Valencia; en el análisis de los resultados, por Óscar Leonardo Ortiz Medina; y en la cooperación en la determinación y el análisis 
de los resultados de concentración y electroforesis en gel, por Javier Adolfo Hernández Fernández.

\section{AgRAdECIMIENTOS}

Los autores expresan su agradecimiento al ingeniero Juan Carlos Calderón, por el suministro del wet white para los ensayos; a la ingeniera Carolina Montefrío, integrante de la empresa MerQuiand, quien aportó la enzima utilizada en cada uno de los ensayos propuestos; de igual manera, a la bióloga María del Pilar Rodríguez Becerra, por su colaboración en la realización de los análisis de Bradford y electroforesis.

\section{REFERENCIAS}

[1] Universidad Nacional del Nordeste, Hipertextos del Área de la biología, 2002. Available at: http://www.biologia.edu.ar/tesis/forcillo/pdf/impacto de los residuos.pdf.

[2] L. A. Artuz, M. S. Matínez, and C. J. Morales, "Las industrias curtiembres y su incidencia en la contaminación del río Bogotá," Isocuanta, vol. 1 (1), pp. 45-53, 2011.

[3] J. Kanagaraj, T. Senthilvelan, R. C. Panda, and S. Kavitha, "Eco-friendly Waste Management Strategies for Greener Environment towards Sustainable Development in Leather Industry: A Comprehensive Review," Journal of Cleaner Production, vol. 89, pp. 1-17, Feb. 2015. https://doi.org/10.1016/j.jclepro.2014.11.013.

[4] J. Barros, "Los trabajadores del cuero que le apuestan al resurgir del río Bogotá." Semana Sostenible, 2018. Available at: https://sostenibilidad.semana.com/impacto/articulo/rio-bogota-curtidores-devillapinzon-y-choconta-dejaron-de-contaminar/39611.

[5] Z. V. Rojas Amaya, Estudio economico-financiero del aprovechamiento de las grasas extraidas del residuo de descarne 'unche' derivado del proceso de curticion en el municipio de Villapinzón, Cundinamarca, Thesis, Universidad Nacional de Colombia, Bogotá D.C., Colombia, 2010. Available at: http://www.bdigital.unal.edu.co/3052/1/790655.2010.pdf.

[6] J. Borges Vilches, Propuesta tecnológica para el curtido de pieles en la tenería "Patricio", Thesis, Universidad Central "Marta Abreu" de Las Villas, Santa Clara, Cuba, 2016. Available at: http://dspace.uclv.edu.cu/bitstream/handle/123456789/7339/JESSICA\%20BORGES\%20VILCHES.pd f? sequence $=1$ \&isAllowed $=y$.

[7] P. Abello Navas, A. Dougall, J. Mandl, and P. Sessa, Reconversión industrial de curtiembres ubicadas en la cuenca Matanza-Riachuelo, Thesis, Instituto Tecnológico de Buenos Aires, Buenos Aires, Argentina, 2012.

Revista Facultad de Ingeniería (Rev. Fac. Ing.) Vol. 28 (52), pp. 59-75. Julio-Septiembre 2019. Tunja-Boyacá, Colombia. L-ISSN: 0121-1129, e-ISSN: 2357-5328, DOI:

https://doi.org/10.19053/01211129.v28.n52.2019.9654 
[8] S. Gorleri, Indumentaria sustentable-indumentaria con cuero reutilizado, Thesis Universidad de Palermo, Buenos Aires, Argentina, 2016. Available at: https://fido.palermo.edu/servicios dyc/proyectograduacion/archivos/4153.pdf.

[9] V. Segarra, Nuevas tecnologías del cuero aplicadas al calzado. Alicante, España: Instituto Tecnológico del Calzado y Coneexas INESCOP, 2008.

[10] M. I. Viracocha Mejía, Evaluación del efecto de tres niveles de sulfato de aluminio y extracto de guarango al 20 \% en la curtición de piel de tilapia roja (Oreochromis sp.), Thesis, Universidad de las Fuerzas Armadas, Santo Domingo de los Tsachilas, Ecuador, 2015.

[11] E. Comes Ribera, Piel vacuna. Barcelona: Cromogenia Linits, 2017, p. 108.

[12] J. P. Orgel, A. Miller, T. C. Irving, R. F. Fischetti, A. P. Hammersley, and T. J. Wess, "The in situ Supermolecular Structure of Type I Collagen," Structure, vol. 9 (11), pp. 1061-1069, 2001. https://doi.org/10.1016/S0969-2126(01)00669-4.

[13] P. Selvakumar, T. C. Ling, A. D. Covington, and A. Lyddiatt, "Enzymatic Hydrolysis of Bovine Hide and Recovery of Collagen Hydrolysate in Aqueous Two-Phase Systems," Separation and Purification Technology, vol. 89, pp. 282-287, Mar. 2012. https://doi.org/10.1016/..seppur.2012.01.046.

[14] R. Paul, J. M. Adzet, M. Brouta-Agnésa, S. Balsells, and H. Esteve, "Hydrolyzed Collagen: A Novel Additive in Cotton and Leather Dyeing," Dyes and Pigments, vol. 94 (3), pp. 475-480, 2012. https://doi.org/10.1016/j.dyepig.2012.02.003.

[15] J. Beltrán Ramírez, Valoración de la innovación tecnológica del proceso de obtención de colágeno a partir de piel de tilapia, Thesis, Universidad Nacional de Colombia, Bogotá D.C., Colombia, 2011.

[16] A. T. Gaona Camacho, and E. A. Pardo Lozano, Implementación de un sistema tecnificado para la valorización de residuos sólidos orgánicos mediante compostaje aplicado a agricultura sostenible en la reserva natural de la sociedad civil Tenasucá, de pedro palo (Tena, Cundinamarca, Colombia), Thesis, Universidad Santo Tomás, Bogotá D.C., Colombia, 2014.

[17] M. F. Jordán Núñez, Obtención de colágeno por hidrólisis alcalina-enzimática del residuo de wet blue en el proceso de curtición, Thesis, Escuela Superior Politécnica de Chimborazo, Riobamba, Ecuador, 2011.

[18] A. C. Nieto Cortés, and A. C. Pedraza Grosso, Evaluación del proceso enzimático para la producción de hidrolizado de colágeno a partir de las virutas de cuero "Wet Blue", Thesis, Universidad Jorge Tadeo Lozano, Bogotá D.C., Colombia, 2017.

[19] S. Jian, T. Wenyi, and C. Wuyong, "Ultrasound-Accelerated Enzymatic Hydrolysis of Solid Leather Waste," Journal of Cleaner Production, vol. 16 (5), pp. 591-597, Mar. 2008. https://doi.org/10.1016/j.jclepro.2006.12.005.

[20] Y. Campo Vera, D. C. Villada Castillo, and J. D. Meneses Ortega, Efecto del pre-tratamiento con ultrasonido en la extracción de peptina contenida en el albedo del Maracuyá," Biotecnología en el Sector Agropecuario y Agroindustrial, vol. 14 (1), pp. 103-109, Jan. 2016. http://dx.doi.org/10.18684/BSAA(14)103-109.

[21] T. Polischuk, E. Buchhamer, J. Navoni, C. Giménez, and E. Villaamil Lepori, Digestión enzimática por ultrasonidos para la determinación de arsénico total en alimentos cocidos: estudio preliminar, 2012. Available at: http://www.frre.utn.edu.ar/iijcyt/clean/files/get/item/2202.

Revista Facultad de Ingeniería (Rev. Fac. Ing.) Vol. 28 (52), pp. 59-75. Julio-Septiembre 2019. Tunja-Boyacá, Colombia. L-ISSN: 0121-1129, e-ISSN: 2357-5328, DOI: https://doi.org/10.19053/01211129.v28.n52.2019.9654 
Evaluación de la hidrólisis enzimática de wet white asistida con ultrasonido para obtener colágeno hidrolizado

[22] J. C. Pestaña Pérez, and J. M. López Rojas, Uso de proteasas a nivel industrial, 2017. Available at: http://www.colmayor.edu.co/archivos/25 uso de proteasas a nivel in mnime.pdf.

[23] C. M. Reyes Mena, Recuperación de colágeno libre de cromo de los residuos sólidos de postcurtición en la industria del cuero, Thesis, Universidad de las Américas, Ambato, Ecuador, 2016.

[24] PMI-Labortechnik, Wisd Laboratory Equipment Ultrasonic Cleaners WiseClean WUC Digital Ultrasonic Cleaner-set, 2010. Available at: http://www.pmi-labortechnik.ch/wiseclean-wuc-digital-ultrasoniccleaner-set-p-74.html?language=en.

[25] H. Gutiérrez Pulido, and R. De la Vara Salazar, Análisis y diseño de experimentos, 2.ㄹ ed., México D.F: McGraw-Hill Interamericana, 2008, p. 564.

[26] M. M. Bradford, "A Rapid and Sensitive Method for the Quantitation of Microgram Quantities of Protein Utilizing the Principle of Protein-Dye Binding," Analytical Biochemistry, vol. 72 (1-2), pp. 248-254, May. 1976. https://doi.org/10.1016/0003-2697(76)90527-3.

[27] J. C. Serrano Gaona, Estandarización de un proceso de extracción de colágeno a partir de los residuos de fileteo de tilapia (Oreochromis sp) y cachama (Piaractus brachypomus), Thesis, Universidad Nacional de Colombia, Bogotá D.C., Colombia, 2011.

[28] O. A. Figueroa, J. E. Zapata, and C. P. Sánchez, "Optimización de la hidrólisis enzimática de proteínas de plasma bovino," Información Tecnológica, vol. 27 (2), pp. 39-52, 2016. http://dx.doi.org/10.4067/S0718-07642016000200006.

[29] J. G. Carrillo Soto, M. Candia Plata, R. E. Lugo Sepúlveda, E. Espinoza Ojeda, and J. A. Noriega Rodríguez, "Evaluación de procedimientos de tinción para el análisis de proteínas por electroforesis (SDS-PAGE)," Invurnus, vol. 8 (1), pp. 19-26, 2013.

[30] A. B. M. Inc., Manual Instruction: Protein Markers-G266, Opti-Protein XL Marker. 2018.

[31] J. VIcéns Otero, A. Herrate Sánchez, and E. Medina Moral. Análisis de varianza (ANOVA), 2005. Available at: http://www.uam.es/departamentos/economicas/econapli/anova.pdf.

[32] C. L. Simbaña Camino, Estudio de propiedades físicas y funcionales de un hidrolizado enzimático de proteína de chocho a escala piloto y su aplicación como fertilizante, Thesis, Escuela Politécnica Nacional, Quito, Ecuador, 2011.

[33] C. Salazar Posada, A. López Padilla, and J. A. Cano Salazar, "Efecto del pH y la temperatura en la hidrólisis enzimática de subproductos de la industria bovina," Revista Lasallista de Investigación, vol. 9 (2), pp. 26-33, 2012.

[34] K. Gómez Lizárraga, C. Piña Barba, and N. Rodríguez Fuentes, "Obtención y caracterización de colágeno tipo I a partir de tendón bovino," Superficies y Vacío, vol. 24 (4), pp. 137-140, 2011.

Revista Facultad de Ingeniería (Rev. Fac. Ing.) Vol. 28 (52), pp. 59-75. Julio-Septiembre 2019. Tunja-Boyacá, Colombia. L-ISSN: 0121-1129, e-ISSN: 2357-5328, DOI:

https://doi.org/10.19053/01211129.v28.n52.2019.9654 